\title{
POLARISING LIGHT BIOMICROSCOPY AND THE RELATION BETWEEN VISUAL ACUITY AND CATARACT
}

\author{
BARBARA K. PIERSCIONEK ${ }^{1,2}$ and ROBERT A. WEALE ${ }^{3,4}$ \\ Bundoora, Australia and London
}

\begin{abstract}
SUMMARY
The use of polarised light in biomicroscopy allows discrimination of lenticular features which are not discernible using standard biomicroscopic techniques. However, just as is true of most brightness-dependent photographic methods testing the effect of filters, past uses of polarised light did not involve any control of illumination. The present study involved the use of polarised light in the photo-biomicroscopy of the anterior segment in cataract patients, the consequential loss in illumination being mimicked with different neutral density filters. This served to distinguish between polarisation and attentuation. The patients were refracted and their corrected visual acuity was recorded. A qualitative scale was drawn up ranking weights of lenticular opacities. Spearman's rank correlation was applied to the photographs obtained with conventional and polarised-light biomicroscopy in turn. Brightness control served to confirm that biomicroscopy with polarised light offers some advantages over conventional methods, and correlates with visual acuity better than is true of conventional biomicroscopy. The Spearman ranks derived from the results obtained with polarised light correlate significantly with the patient's visual acuity. It is concluded that polarised light biomicroscopy, in conjunction with the standard examination, can improve the diagnosis of cataract and assist in relating it to a patient's visual acuity.
\end{abstract}

Slit lamp biomicroscopy has been a standard clinical technique throughout this century and is the major method used for observing and assessing cataracts. However, the image seen often fails to correlate with

From: ${ }^{1}$ Department of Electronic Engineering, Latrobe University, Bundoora, Victoria 3083, Australia; ${ }^{2}$ National Vision Research Institute, Carlton, Victoria 3053, Australia; ${ }^{3}$ Age Concern Institute of Gerontology, King's College London, UK; ${ }^{4}$ Eye Department, University College Hospital, London, UK.

Correspondence to: Robert A. Weale, Age Concern Institute of Gerontology, King's College London, Cornwall House Annexe, Waterloo Road, London SE1 8WA, UK. the patient's visual acuity or function. Indeed the relationship between the alterations in the structural proteins, the increase in light scatter associated with conventional biomicroscopy, and the capacity of visual function is not simple and has yet to be defined.

It has been found clinically that visual acuity can remain high in spite of an age-related increase in light scatter. ${ }^{1}$ The reason for this inconsistency is that the scattered light has not been analysed. The progressive insolubilisation of proteins with age is said to cause density fluctuations which scatter light and impair vision. ${ }^{2}$ However, what the slit lamp reveals as scattered light may not necessarily be diffuse scatter resulting from variations of refractive index within fibres or from small inhomogeneities due to protein aggregation, but may also contain components of reflected light. It has been pointed out $^{3,4}$ that a substantial part of apparently scattered light is due to specular reflections which may not degrade vision. If the lens is examined using biomicroscopy, with the incident light polarised and the image viewed with a crossed analyser over the eyepiece, a significant proportion of the scattered light seen conventionally (in which incident light is unpolarised) is extinguished. Reflected light remains polarised while diffusely scattered light becomes depolarised. Therefore, a proportion of what is referred to as scattered light may in fact be reflected light.

Briefly, when an eye is illuminated by planepolarised light and viewed or photographed through a crossed polariser, a dark cross appears. ${ }^{3,5-8}$ The relative contributions of corneal birefringence ${ }^{7,9-13}$ and refraction ${ }^{14}$ remain to be decided. Fig. 1a shows the dark cross which is absent in conventional natural, illumination (Fig. 1b). The appearance of the cross is contingent on near-perpendicular illumination and viewing. This may be inconvenient under clinical conditions, as when strabismus is 


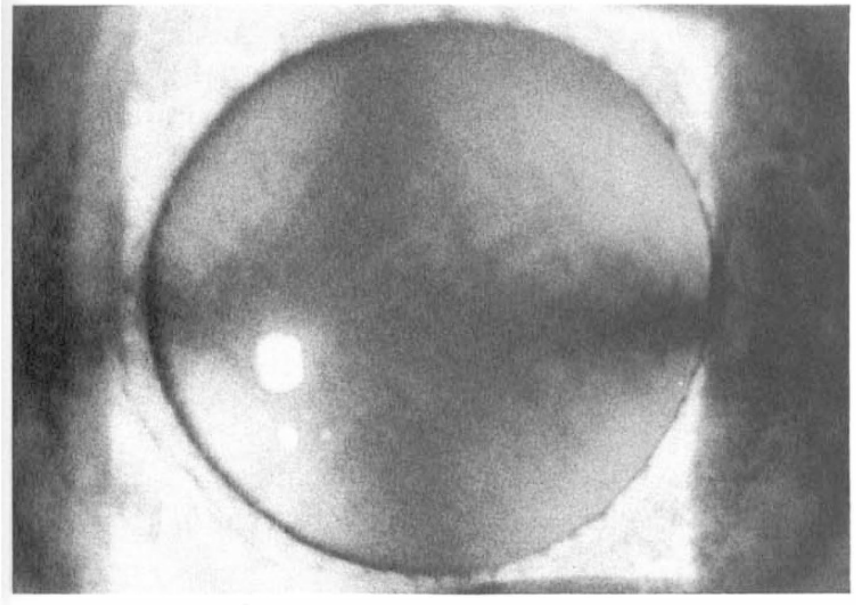

(a)

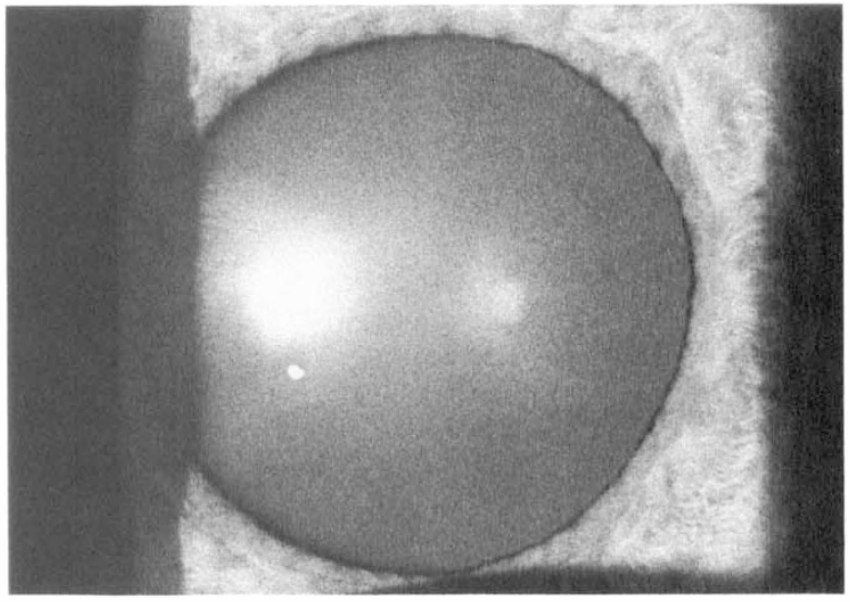

(b)

Fig. 1. Photographs of the lens taken using (a) PLB (polarised light biomicroscopy) and (b) standard biomicroscopy matched to (a) for brightness attentuation. The lens shown is from an eye with VA of 0.67 (subject 12, Table II).

present, or when the lens is preferably viewed at different angles. When scattering loci are present within the confines of the cross, depolarisation of the incident light will cause such opacities to become visible. In particular, opalescence, generally associated with nuclear changes, can also be reduced, if not altogether eliminated, with polarised light biomicroscopy (PLB). This is observed regardless of whether the eye is examined in focal or oblique illumination ${ }^{4}$ and therefore renders polarised light clinically useful.

The original study, ${ }^{4}$ in which opacities which were not visible using standard biomicroscopy could be seen clearly using PLB, did not take into account (1) the role which may have been played by the reduction of illumination resulting from the use of crossed polarisers, and (2) the connection between PLB images and (corrected) visual acuity. It should be emphasised that, as far as we know, no study involving ocular black-and-white photography with a variety of, say, chromatic filters, has been supported by the application of this important control of the effect of altered illumination.

Accordingly, we sought to break fresh ground, and test whether PLB produced qualitative or merely quantitative differences in slit lamp photography, and also whether it could help to improve the correlation between slit lamp image and visual acuity.

\section{METHODS}

Subjects without retinal pathology ( 4 men, 8 women; age range 56-73 years, mean 63 (5.09) years) were selected from a private practice in Melbourne, Australia. Lenses from one eye of 9 and both eyes of 3 individuals were used in the study; the former group had implants in the eye not photographed. They were refracted, and their corrected visual acuity (VA) was determined to the nearest letter.
After pupil dilation (with 1 drop of $0.5 \%$ tropicamide) lenses were photographed using a Shin Nippon SL202 photo-slit lamp. For the purpose of this study two sheet polarisers were used (Lee linear type polarising camera filters). One was placed with its polarisation axis horizontal between the illuminating source and the mirror reflecting the slit beam into the eye; the other, with its axis vertical, covered the whole of the objective. It is to be stressed that, following the previous study by Weale, ${ }^{4}$ no retroillumination was employed as this would greatly curtail light on account of the reflecting properties of the fundus oculi.

After photography through crossed polarisers these were removed and the lenses were photographed at various known relative intensities. These involved three neutral density filters (Schott glass filters) - $1.6,2.0$ and 2.4 (i.e. transmitting $2.5 \%, 1 \%$ and $0.4 \%$ of the incident light) - in turn, with flash intensity set at 4 , and, except for the first 4 cases, without any filters and flash intensity set at 2 . These photographs served the purpose of providing an approximate match in illuminating intensity. The range of neutral density filters had been determined previously in a trial run. Ilford HP5 was used and developed to 800 ASA with Kodak Tmax developer for 8.5 minutes (with 1 minute agitation) at $20.5^{\circ} \mathrm{C}$.

In order to compare results obtained by the two methods, we set up a scale of weights for the various photographic appearances (Table I), and calculated correlations with VA for each in turn. Also, the usual plusses and minuses given with VA assessments were quantified by dividing the step between any two lines by the number of letters in neighbouring lines, and adding or subtracting the appropriate value. For example, the line for VA = 1 contains seven letters, that for $0.67 \mathrm{six}$, and that for 0.5 five. Thus VA = $0.5++$ would be translated into $0.5+[(0.67-0.5) / 6]$ $\times 2=0.557$. Similarly VA $=1-$ becomes $1-[(1-$ 
Table I. Weight factors used in the ranking process

\begin{tabular}{lc}
\hline Type of opacity & Weight \\
\hline Nil & 1 \\
Coronal cataract & 1 \\
A few punctate opacities & 2 \\
Many punctate opacities & 3 \\
Peripheral striae & 4 \\
Cortical striae & 5 \\
Haze & 6 \\
Central striae & 6 \\
Central (axial) opacity & 7 \\
\hline
\end{tabular}

$0.67) / 7] \times 2=0.91$. All the results were converted to the LogMAR scale $;^{15}$ note that when VA $=1$, Log MAR $=0$, and when VA $=0.1, \operatorname{LogMAR}=-1$. Where more than one weight factor was pertinent, the larger was used. Spearman's rank correlation coefficients were calculated for comparisons between LogMAR values and the appropriate data from Table I for each patient; averages were used for the 3 patients where results for two eyes were obtained.

\section{RESULTS}

The details of the 12 subjects are shown in Table II. In all cases where VA was $0.8++(6 / 7 / 5++, 20 / 25++$; $\operatorname{LogMAR}=-0.075)$ or better the images seen through the crossed polarisers showed a less dense lenticular haze and enhanced visibility of details such as punctate opacities (Fig. 2). In the photograph in Fig. 2a, taken using PLB, there is a heavy sprinkling of punctate opacities, almost crystal-like in appearance, particularly concentrated in the anterior polar region. They are invisible in Fig. $2 b$, taken through neutral density filters matched for attenuation of brightness. Here only a few of the larger opacities in the peripheral regions are obvious. Although all these punctate opacities scatter light, the smaller central ones seen in Fig. 2a are masked by a veil of light reflected from the anterior surface of the lens and possibly also from internal lenticular structures.

In cases with greater visual impairment, in which opacities may not be superficial and hence may be difficult to see, these become distinguishable using PLB. Photographs taken from an eye with a VA of $0.67-(6 / 9-, 20 / 30-; \operatorname{LogMAR}=-0.196)$ are shown in Fig. 3. Fig. 3a, the PLB image, shows a conglomeration of denser, larger opacities than those seen in Fig. 2a. These are located in deeper regions of the lens and hence have indistinct borders giving an overall stippled appearance. In particular there is a hazy patch close to the centre of the lens which would contribute to the loss of acuity. The image viewed through matching filters (Fig. 3b) appears to be quite clear with only a slight opacification evident in the 7 o'clock position. If the opacities in Figs. 2a and 3a are to be identified with retrodot, then it is worth considering whether this condition is as rare as has been thought.

Another example is given in Fig. 4 taken from an eye with $0.67-(6 / 9-, 20 / 30-)$ vision. The image photographed through PLB (Fig. 4a) shows a greater number of spoke opacities and provides" a better indication of how far into the inner regions of the lens they extend. The true size of these features is not visible when viewing through matched filters (Fig. 4b). In addition, the sutural cataract and surrounding haze are enhanced in Fig. 4a.

The Spearman rank analysis gives a correlation coefficient of 0.5524 (NS) between VA and weight for conventional biomicroscopy, and one of 0.6521 $(p<0.02)$ for the arrangement with polarised light.

\section{DISCUSSION}

This study validates and extends the previous work on PLB. ${ }^{4}$ In particular, it indicates that PLB is a useful supplement to the conventional biomicroscopic examination. It facilitates the detection of lenticular anomalies which would otherwise remain undetected. In the presence of good VA it offers earlier alerts than the conventional method and may therefore be the method of choice in preventive therapy, if any. It may also resolve the clinical

Table II. Subject parameters and photographic results

\begin{tabular}{|c|c|c|c|c|c|c|c|}
\hline $\begin{array}{l}\text { Subject } \\
\text { and gender }\end{array}$ & $\begin{array}{l}\text { Age } \\
(\mathrm{yr})\end{array}$ & $\begin{array}{c}\mathrm{VA} \\
(-\mathrm{LogMAR})\end{array}$ & Eye & Conventional viewing & Weight & PLB & Weight \\
\hline $1 \mathrm{M}$ & 56 & 0 & $\mathbf{R}$ & Haze & 6 & Minor punctate opacities & 2 \\
\hline $1 \mathrm{M}$ & 56 & 0.022 & $\mathrm{~L}$ & Haze and a few punctate opacities & 6 & Multiple punctate opacities & 3 \\
\hline $2 \mathrm{M}$ & 59 & 0.045 & $\mathrm{~L}$ & Peripheral striae & 4 & Nothing significant & 1 \\
\hline $3 \mathrm{M}$ & 61 & 0.075 & $\mathbf{R}$ & Haze & 6 & Diametric streak & 5 \\
\hline $4 \mathrm{~F}$ & 73 & 0.175 & $\mathrm{~L}$ & Paracentral radial striae & 4 & Ditto accentuated and axial opacity & 7 \\
\hline $5 \mathrm{~F}$ & 61 & 0.196 & $\mathrm{~L}$ & Haze & 6 & Deep central opacity & 7 \\
\hline $6 \mathrm{M}$ & 67 & 0.196 & $\mathrm{~L}$ & Cortical striae & 5 & Cortical strieae, central streaks & 6 \\
\hline $7 \mathrm{~F}$ & 58 & 0.196 & $\mathbf{R}$ & Cortical striae, suture clefts & 5 & Cortical striae, axial opacity & 7 \\
\hline $7 \mathrm{~F}$ & 58 & 0.543 & $\mathrm{~L}$ & Haze, cortical and central opacities & 7 & Cortical striae, central opacities & 7 \\
\hline $8 \mathrm{~F}$ & 64 & 0.258 & $\mathrm{~L}$ & Haze & 6 & Star-shaped cortical opacity & 5 \\
\hline $9 \mathrm{~F}$ & 70 & 0.258 & $\mathbf{R}$ & Haze & 6 & Central opacities & 7 \\
\hline $9 \mathrm{~F}$ & 70 & 0.3 & $\mathrm{~L}$ & Haze & 6 & Central opacities & 7 \\
\hline $10 \mathrm{~F}$ & 65 & 0.3 & $\mathbf{R}$ & Cortical and nuclear opacities & 7 & Cortical and nuclear opacities & 7 \\
\hline $11 \mathrm{~F}$ & 59 & 0.3 & $\mathbf{R}$ & Haze and coronal cataract & 6 & Coronal cataract, central opacities & 7 \\
\hline $12 \mathrm{~F}$ & 62 & 0.175 & $\mathbf{R}$ & Haze & 6 & Axial opacities & 7 \\
\hline
\end{tabular}

$\mathrm{M}$, male; F, female; $\mathrm{R}$, right; $\mathrm{L}$, left. 


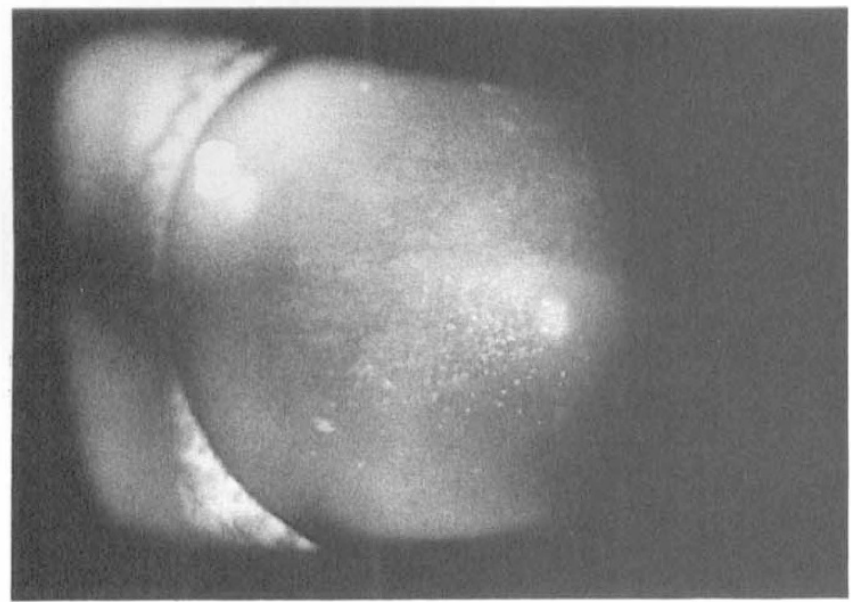

(a)

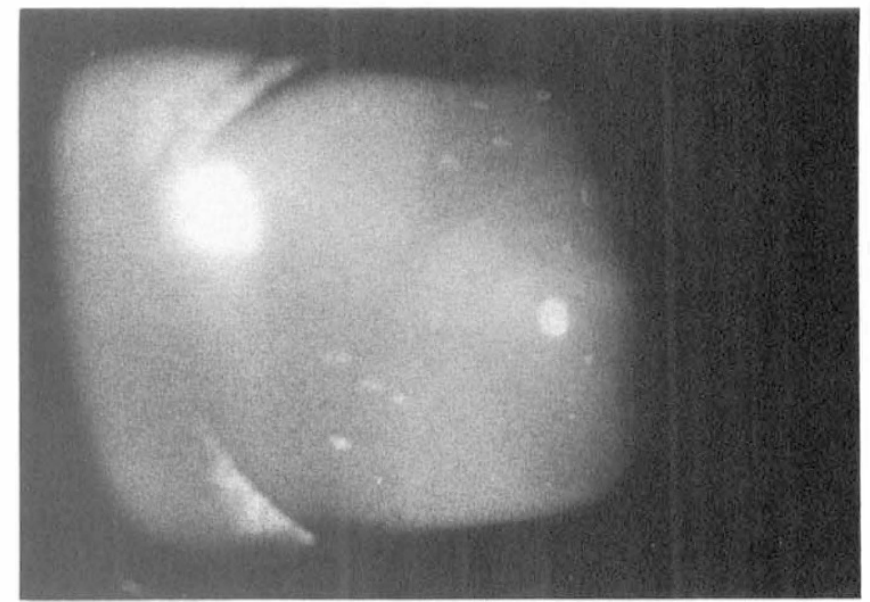

(b)

Fig. 2. Photographs of the lens from an eye with VA of 1- (subject 1, Table II) taken using (a) PLB and (b) standard biomicroscopy matched to Fig. $2 a$ for brightness attentuation.

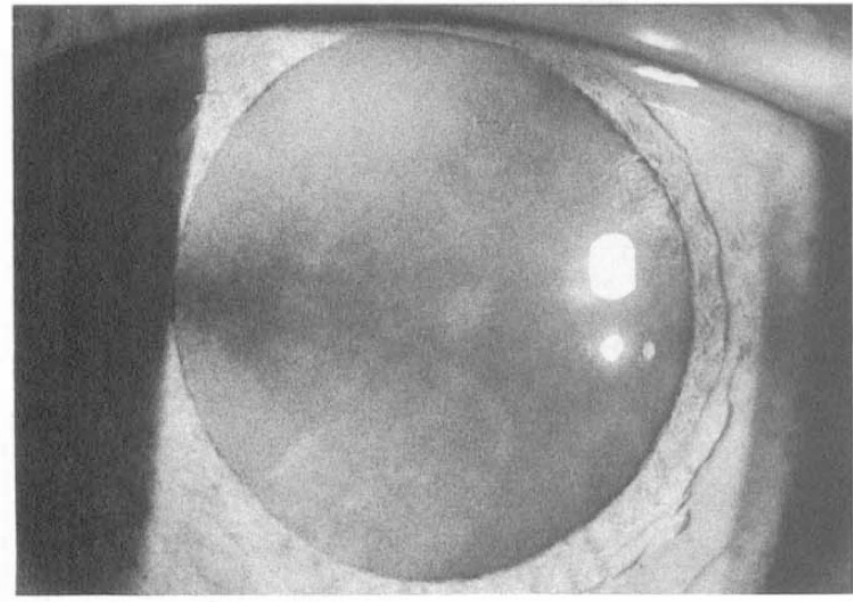

(a)

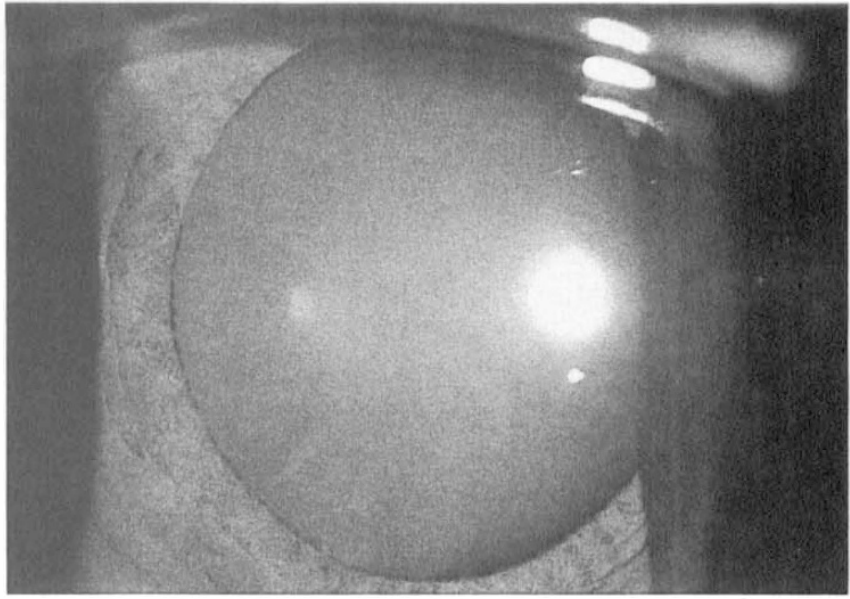

(b)

Fig. 3. Photographs of the lens from an eye with VA of $0.67-$ (subject 5, Table II) taken using (a) PLB and (b) standard biomicroscopy matched to Fig. 3 a for brightness attenuation.

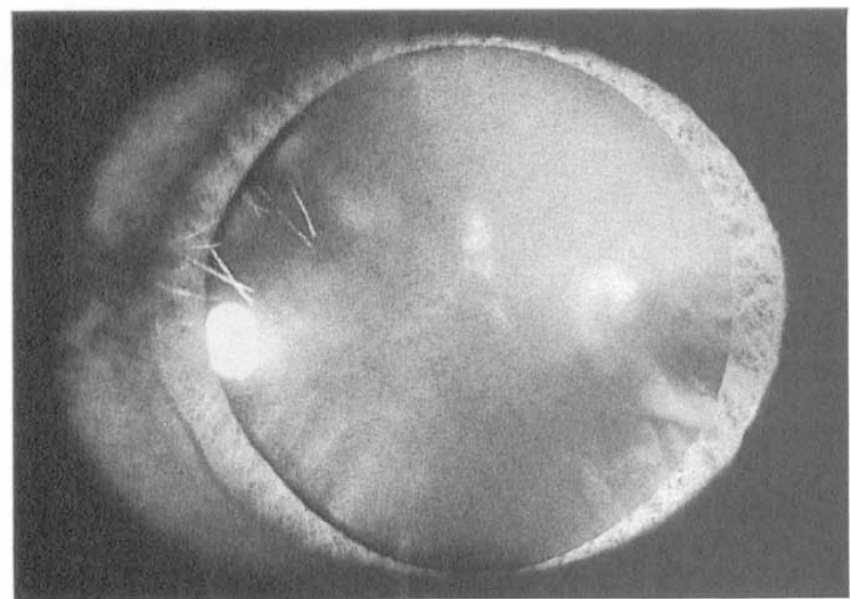

(a)

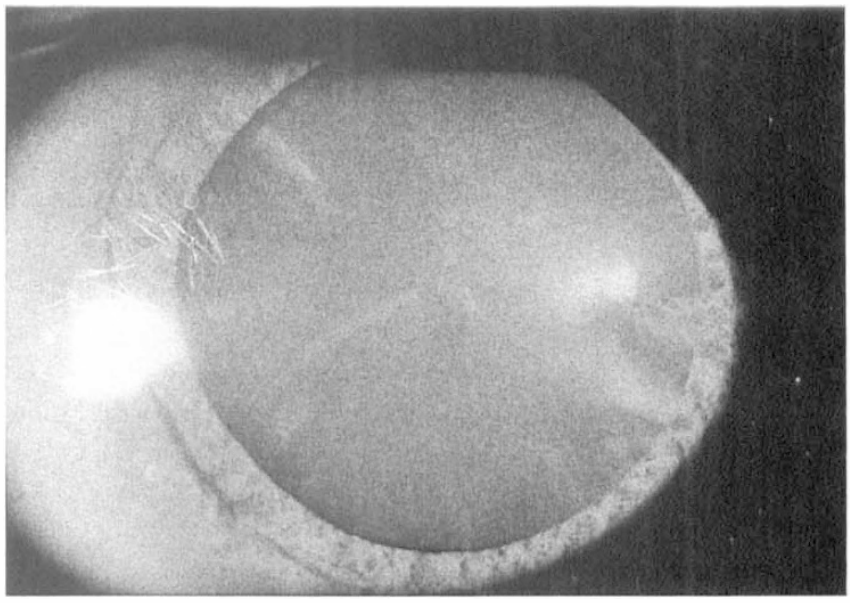

(b)

Fig. 4. Photographs of the lens from an eye with VA of 0.67- (subject 7, Table II) taken using (a) PLB and (b) standard biomicroscopy matched to Fig. 4 a for brightness attenuation. 
paradox created by the inconsistency of a patient's VA with biomicroscopic findings. A comparison of Fig. $2 b$ with either Fig. $3 b$ or $4 b$ indicates that conventional biomicroscopy could not predict VA: the images seen in Figs. 2b, 3b and 4b would suggest little difference in VA between those eyes. However, with PLB it becomes evident that there are dense central opacities in the eyes with lower VA (Figs. 3a and $4 a$ ) while the opacities in Fig. 2a are punctate and widely scattered, and hence less detrimental to vision.

Moreover, attention should be drawn to some epidemiological aspects. Understandably, current classifications of cataract are based on appearance. $^{16,17}$ PLB shows that appearances can be deceptive, and that it may be necessary to distinguish, for example, between seeming nuclear anomalies noted in normal viewing but disappearing in PLB, namely structures which reflect but do not scatter light and are therefore less likely to degrade vision. This applies also to various studies of risk factors $^{18-20}$ in which the prevalence of nuclear cataracts may be singled out, as happens for example in connection with smoking. The question then arises whether the odds ratio is changed when PLB is used. Finally, Thompson $e t$ al. ${ }^{21}$ have recently published a longitudinal study which showed that there is a statistically significant link between nuclear cataract and mortality. Here again, the use of PLB may change the statistics underlying the conclusions reached.

The authors thank Mr K. Zagoritis for use of his clinical facilities and access to volunteers. Thanks are also due to the volunteers for their helpful co-operation. B.K.P. is supported by the N.H.M.R.C. (R. D. Wright fellowship). R.A.W. gratefully acknowledges partial support from Research into Ageing.

Key words: Cataract, Cataract classification, Eye lens, Lens photography, Polarised light, Slit lamp.

\section{REFERENCES}

1. Sigelman J, Trokel SL, Spector A. Quantitative biomicroscopy of lens light backscatter. Arch Ophthalmol 1974;92:437-42.
2. Benedek G. Theory of transparency of the eye. Appl Optics 1971;10:459-73.

3. Weale RA. Real light scatter in the human crystalline lens. Graefes Arch Clin. Exp Ophthalmol 1986; 224:463-6.

4. Weale RA. New method for visualising discontinuities in the crystalline lens. Br J Ophthalmol 1986;70:925-30.

5. Cogan DG. Some ocular phenomena produced with polarised light. Arch Ophthalmol 1941;25:391-400.

6. Stanworth A, Naylor EJ. The polarisation optics of the isolated cornea. Br J Ophthalmol 1950;34:210-1.

7. Stanworth A, Naylor EJ. Polarisation light studies of the cornea. I. The isolated cornea. J Exp Biol 1953;30:160-3.

8. Cope WT, Wolbarsht ML, Yamanashi BS. The corneal polarisation cross. J Opt Soc Am 1978;68:1139-41.

9. Maurice DM. The structure and transparency of the cornea. J Physiol 1957;136:263-86.

10. Post D, Gurland JE. Birefringence of the cat cornea. Exp Eye Res 1966;5:286-95.

11. Kaplan D, Bettelheim FA. On the birefringence of bovine cornea. Exp Eye Res 1972;13:219-26.

12. Wang TJY, Bettelheim FA. Comparative birefringence of cornea. Comp Biochem Physiol 1975;51A:89-94.

13. Bour LJ, Cardozo NJL. On the birefringence of the living human eye. Vision Res 1981;21:1413-21.

14. Pierscionek BK. Explanation of isogyre formation by the eye lens. Ophthalmic Physiol Optics 1993;13:91-4.

15. Bailey IL, Lovie JE. New design principles for visual acuity letter charts. Am J Optom 1976;53:740-5.

16. Sparrow JM, Bron AJ, Brown NAP, Ayliffe W, Hill AR. The Oxford clinical cataract classification and grading system. Int Ophthalmol 1986;9:207-25.

17. Chylack LT Jr, Wolfe JK, Singer D, Leske MC, Bullimore MA, Bailey IL, Friend J, McCarthy D, Wu S-Y, for the Longitudinal Study of Cataract Study Group. The Lens Opacities Classification System III (LOCS III). Arch Ophthalmol 1993;111:831-6.

18. Flaye DE, Sullivan KN, Cullinan TR, Silver JH, Whitelocke RAF. Cataracts and cigarette smoking. Eye 1989;3:379-84.

19. West S, Munoz B, Emmett EA, Taylor HR. Cigarette smoking and risks of nuclear cataract. Arch Ophthalmol 1989;107:1166-9.

20. The Italian-American Cataract group. Risk factors for age-related cortical, nuclear and posterior subcapsular cataracts. Am J Epidemiol 1991;133:541-53.

21. Thompson JR, Sparrow JM, Gibson JM, Rosenthal AR. Cataract and survival in an elderly non diabetic population. Arch Ophthalmol 1993;111:675-9. 